\title{
Functional properties of an ensemble of candidate germline-encoded precursors of the anti-MPER antibody 4E10
}

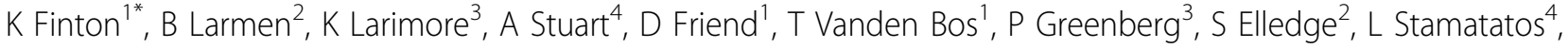 \\ R Strong ${ }^{1}$
}

From AIDS Vaccine 2012

Boston, MA, USA. 9-12 September 2012

\section{Background}

We have previously engineered computationally-designed 'epitope-scaffold' constructs for the broadly neutralizing, MPER-specific antibody 4E10, consisting of the epitope grafted as a structural unit onto non-HIV scaffold proteins for optimal presentation during immunization. 4E10 epitope-scaffolds display dissociation constants for mature 4E10 ranging down to picomolar values and can elicit epitope-specific responses during immunization. Sera from immunized animals failed to potently neutralize HIV, at least partially due to differences between human and nonhuman germline repertoires. Successful use of epitopescaffolds as vaccine immunogens will require optimizing interactions with both the mature antibody target and appropriate precursors, while preserving or generating neutralization potency during maturation.

\section{Methods}

Since a unique germline-encoded precursor sequence for 4E10 cannot be unambiguously assigned, we have generated an ensemble of the twelve likeliest candidates in order to study their functional and recognition properties. Interaction parameters between mature and candidate germline-encoded precursor (CGP) antibodies for engineered epitope-scaffolds, peptides, membrane components and HIV proteins were determined in surface plasmon resonance analyses and three-dimensional structures of free and ligand-complexed forms were determined by $\mathrm{x}$-ray crystallography. Neutralization potencies were determined and polyspecificity and autoreactivity were analyzed with large-scale peptide arrays.

\section{Results}

Unlike other anti-HIV CGPs, which display negligible affinities for HIV-related ligands, 4E10 CGPs display affinities for epitope-scaffolds which, while 1,000- to 100,000-fold weaker, still reach into the nanomolar range. Structural studies show remarkable conservation of recognition mechanisms while functional studies show retention of anti-HIV activity. Polyspecificity of both mature and CGP forms is very limited, but the significant autoreactivity of mature 4E10 appears directed to specific targets.

\section{Conclusion}

The functional gap between mature 4E10 and its CGPs is narrower than in other HIV-related systems. Strategies based on our results can be proposed to generate matureand CGP-specific epitope-scaffolds for use in prime-boost vaccinations to target specific CGPs with desirable functional properties while potentially avoiding autoreactivity.

\section{Author details \\ ${ }^{1}$ Fred Hutchinson Cancer Research Center, Seattle, WA, USA. ${ }^{2}$ Harvard Medical School, Boston, MA, USA. ${ }^{3}$ University of Washington, Seattle, WA, USA. ${ }^{4}$ Seattle Biomedical Research Institute, Seattle, WA, USA.}

Published: 13 September 2012

doi:10.1186/1742-4690-9-S2-P344

Cite this article as: Finton et al.: Functional properties of an ensemble of candidate germline-encoded precursors of the anti-MPER antibody 4E10. Retrovirology 2012 9(Suppl 2):P344.

${ }^{1}$ Fred Hutchinson Cancer Research Center, Seattle, WA, USA

Full list of author information is available at the end of the article

C 2012 Finton et al; licensee BioMed Central Ltd. This is an Open Access article distributed under the terms of the Creative Commons 